\title{
Systematic tracking of altered modules identifies the key biomarkers involved in chronic lymphocytic leukemia
}

\author{
XIN LU $^{1}$, ZHEN WU $^{1}$, XUE-YING ZHAO $^{1}$, CHUN-FENG LI $^{1}$ and SHI-FENG KAN ${ }^{2}$ \\ ${ }^{1}$ Department of Blood Transfusion, Second Hospital of Shandong University, Jinan, Shandong 250033; \\ ${ }^{2}$ Department of Laboratory Medicine, Qilu Hospital of Shandong University, Jinan, Shandong 250012, P.R. China
}

Received May 24, 2018; Accepted November 27, 2018

DOI: $10.3892 / \mathrm{ol} .2018 .9812$

\begin{abstract}
Key genes in chronic lymphocytic leukemia (CLL) were investigated through systematically tracking the dysregulated modules from protein-protein interaction (PPI) networks. Microarray data of normal subjects and CLL patients recruited from ArrayExpress database were applied to extract differentially expressed genes (DEGs). Additionally, we re-weighted the PPI network of normal and CLL conditions by means of Pearson's correlation coefficient (PCC). Furthermore, clique-merging method was applied to extract the modules and then the altered modules were screened out. The intersection genes were selected from miss and add genes in the altered modules. The common genes were screened from the intersection genes and DEGs in CLL. A total of 734 DEGs were screened by statistical analysis. In this investigation, there were 1,805 and 703 modules in normal as well as disease PPI network. In addition, 875 altered modules were obtained which included 145 miss genes, 353 add genes and 85 intersection genes. Finally, in-depth analysis revealed 9 mutual genes between the intersection genes and DEGs in CLL. Our analysis revealed several key genes associated with CLL by systematically tracking the dysregulated modules, which might be candidate targets for diagnosis and management of CLL.
\end{abstract}

\section{Introduction}

Fundamental units formed by interacting proteins have been demonstrated to be responsible for implementing important biological processes within cells (1). The identification of protein complexes (modules or pathways) contributes to understanding the complex formation and the higher level organization of cells, and the dysregulation of protein complexes

Correspondence to: Dr Shi-Feng Kan, Department of Laboratory Medicine, Qilu Hospital of Shandong University, 115 Wenhuaxi Road, Jinan, Shandong 250012, P.R. China

E-mail: ksf5602@163.com

Key words: chronic lymphocytic leukemia, protein-protein interaction network, altered modules, differentially expressed genes might be associated with the development of disease. With the advance of microarray techniques, a large number of physical interactions have been cataloged from organisms, fueling the development of computational algorithms for systematically mining protein complexes from the protein-protein interaction (PPI) network. Despite the significant progress in the aspect of extracting protein complexes from the PPI networks over the last few years, computational methods are seriously constrained by noise in current PPI data $(2,3)$. Several computational methods have been developed to predict modules from networks relying on graph clustering, and clique finding, such as MCODE (4), CMC (5) and STM (6). In addition, the identification of dysregulated modules from condition-specific networks will promote the process to reveal the pathogenesis of disease. Recently, a systematic method for uncovering dysregulated modules has been developed by integrating PPI information and condition-specific gene expression profile (7).

Chronic lymphocytic leukemia (CLL) is the most common type of hematopoietic malignancies in adults (8). Nowadays, more and more patients are diagnosed at early-stage, likely because of using routine blood tests as well as the widespread availability of flow cytometry (9-11). However, the treatment of CLL remains a challenge (12). A previous study demonstrated that TL1A could be considered as a negative regulator of leukemic cell proliferation, that may influence the physiopathology and clinical outcome of CLL at an early-stage (13). Moreover, researchers indicated that the inhibition of JAK was a potentially useful new pharmacological approach to CLL treatment (14). However, the underlying mechanisms of CLL have yet to be fully elucidated. Systematic tracking dysregulated module behavior across specific conditions is critical.

In this investigation, we proposed a straightforward but systematic method to identify and compare the modules across normal and CLLs through integrating PPI data and specific gene expression profile of CLL. The results of this study will be critical to reveal the molecular mechanism of this disease and develop promising therapies.

\section{Materials and methods}

Data recruitment and preprocessing. Here we selected the microarray expression profile of E-GEOD-2466 (15) CLL from ArrayExpress database to perform our analysis. The gene expression profile numbered E-GEOD-2466 which was based 
on the platform of A-AFFY-1 - Affymetrix GeneChip Human Genome U95Av2 [HG_U95Av2] and A-AFFY-9 - Affymetrix GeneChip Human Genome U95A [HG_U95A] was downloaded. E-GEOD-2466 comprised a total of 111 samples, including 100 genetically well-characterized B-CLL samples and 11 normal control samples. Finally, we obtained the probe annotation data for subsequent analysis.

With the goal of eliminating the influence of non-specific hybridization, the expresso function from the Affy package was used to obtain the probe data. Moreover, background correction was conducted by means of robust multiarray average (RMA) (16), quantiles (17) was employed to standardize the data, MAS was utilized to implement perfect match (PM) and mismatch (MM) correction (18), medianpolish (16) was performed to summarize the probe data, followed by the filtration of probe data by means of featureFilter function. Ultimately, we obtained 20,102 genes from the probe set.

Detection of differentially expressed genes (DEGs). The DEGs between the two groups were extracted relying on limma package (19). Empirical Bayes (eBayes) (20) as well as a false discovery rate (FDR) (21) calibration of P-values were implemented using ImFit function. The genes were extracted if these genes met the following conditions: $\mid \log \mathrm{FCl} \geq 2, \mathrm{P}<0.01$.

PPI network construction. In this study, we downloaded all PPI data with combine-score from the STRING database (22). The protein ID and gene symbols were transformed and then the repeated ones were removed. We selected the interaction relations with combined-score $\geq 0.65$ to construct the PPI sub-network.

Gene interactions re-weighted using Pearson's correlation coefficient (PCC). Gene interactions of the PPI sub-network of CLL and normal subjects were re-weighted by PCC. PCC can evaluate the probability of two co-expressed genes, where value ranged from -1 to +1 (23). Moreover, the absolute value of PCC of each edge was defined as the value of the interaction of the PPI sub-network. In addition, we defined PCC of every gene pair as the weight value of the edge.

Uncovering modules from the sub-network of PPI.Clique-merging, an approach used to identify the modules, is similar to the previous algorithms for detecting complexes from the PPI networks $(2,5)$. Herein, we used the clique-merging to extract the modules from PPI sub-networks. In detail, we discovered all maximal cliques from the network of the normal and disease conditions using fast depth-first approach. Subsequently, the maximal cliques having nodes $\geq 5$ were selected. Last we arranged them in descending order according to the score of a clique. This score was its weighted interaction density (WID). According to the WID, we sorted these cliques.

A lot of maximal cliques may be overlapped. Hence, these lapped cliques should be abandoned to decrease size or merged into a bigger sub-graphs. In this study, the weighted inter-connectivity between two cliques was employed to determine whether these two lapped cliques were combined or not. Herein, t0 (a predefined overlap cut-off criteria) $=0.5$ and tm (a predefined $\mathrm{m}$ cut-off criteria) $=0.25$ were selected to be the threshold in the current study.

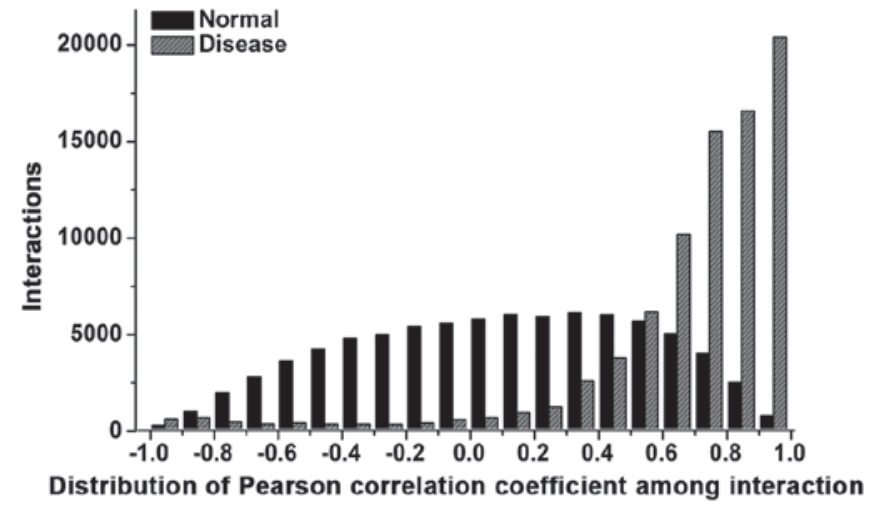

Figure 1. Interaction distribution in normal and CLL groups. CLL, chronic lymphocytic leukemia.

Identification of altered modules. The modules were respectively extracted from the PPI networks of normal controls and CLL. For each module in the normal and disease conditions, we calculated the modules correlation density (MCD) based on the PCC of gene-gene interactions in PPI network. MCD was measured as:

$$
d_{c c}\left(S_{i}\right)=\frac{\sum_{p, q \in S_{i}} P C C((p, q), N)}{\left(\begin{array}{c}
\left|S_{i}\right| \\
2
\end{array}\right)} .
$$

The pairs of the disrupted or dysregulated modules were evaluated through determining the module as a maximum weight bipartite matching (24). A similarity graph $\mathrm{M}=(\mathrm{Vm}, \mathrm{Em})$ was constructed, where $\mathrm{Vm}=\{\mathrm{SUT}\}$, and $\mathrm{Em}=\mathrm{U}\left\{\left(\mathrm{S}_{\mathrm{i}}, \mathrm{T}_{\mathrm{j}}\right) \mathrm{J}\left(\mathrm{S}_{\mathrm{i}}, \mathrm{T}_{\mathrm{j}}\right) \geq \mathrm{tJ}\right.$, $\Delta \operatorname{CC}\left(\mathrm{S}_{\mathrm{i}}, \mathrm{T}_{\mathrm{j}}\right) \geq \delta$, whereby $\mathrm{J}\left(\mathrm{S}_{\mathrm{i}}, \mathrm{T}_{\mathrm{j}}\right)=\left|\mathrm{S}_{\mathrm{i}} \cap \mathrm{T}_{\mathrm{j}} / / \mathrm{S}_{\mathrm{i}} \cup \mathrm{T}_{\mathrm{j}}\right|$ was the Jaccard similarity $(\mathrm{J})$ and $\Delta \mathrm{CC}\left(\mathrm{S}_{\mathrm{i}}, \mathrm{T}_{\mathrm{j}}\right)=\left|d_{c}\left(S_{i}\right)-d_{c}\left(T_{i}\right)\right|$ was the differential correlation density $(\Delta C C)$ between $S_{i}$ and $T_{j}$ (7). In our study, the altered modules with $t J \geq 2 / 3$ as well as $\Delta \mathrm{CC} \geq 0.05$ were considered as the disrupted modules.

Gene composition of altered modules. With the goal of better understanding the differences of the altered modules in the two groups, we screened out the missed and added genes. Significantly, the common part of the missed as well as added genes was also identified. Moreover, in order to obtain the relevant disease genes, we also took the intersection of the DEGs and the intersection genes.

\section{Results}

Detection of DEGs. In our study, a total of 8,630 genes were screened out for subsequent analysis. Using the threshold of $\mid \log \mathrm{FCl}>2$, and a FDR $<0.01$, a total of 734 genes were identified to be differentially expressed in CLL.

Disruptions in CLL PPI network. Under the combined-score $\geq 0.65$, the PPI sub-networks displayed equal numbers interactions $(40,576)$ and nodes $(6,068)$. Their mean average scores were respectively 0.320 and 0.698 in the normal and disease network. The result of PCC distribution in the two networks is shown in Fig. 1. Differences were observed in the PCC score distribution (-1 to 1) of the two networks. Moreover, the count of the interactions in the normal network was higher than that 
Table I. Properties of normal and disease modules.

\begin{tabular}{lccccc}
\hline & & \multicolumn{3}{c}{ Correlation } \\
\cline { 4 - 5 } Module set & No. of modules & Average module size & \multicolumn{1}{c}{ Max } & Avg & \multirow{2}{*}{ Min } \\
\hline Normal & 1,805 & 19.32798 & 0.5186083 & 0.06900261 & -0.1587027 \\
Disease & 703 & 43.46373 & 0.573716 & 0.0379185 & -0.1173073 \\
\hline
\end{tabular}

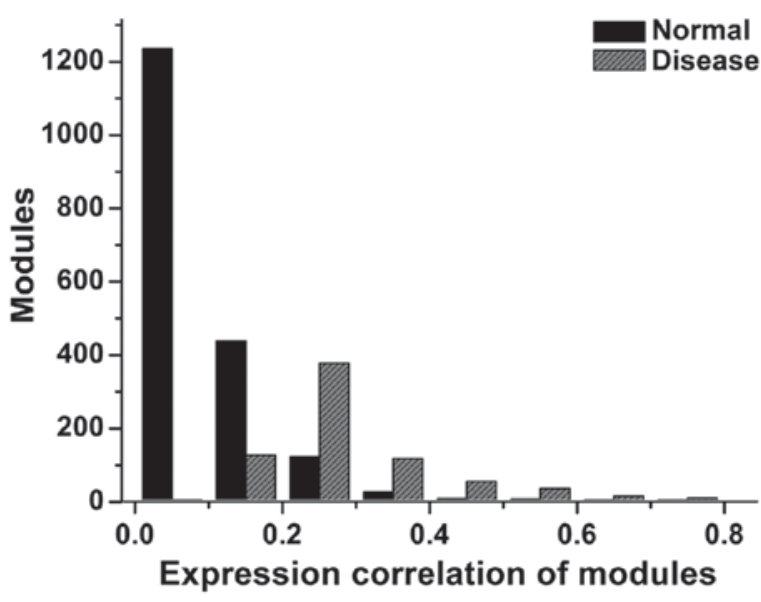

Figure 2. Distribution of the correlation-wise values of modules in normal and CLL group. CLL, chronic lymphocytic leukemia.

in the CLL network when the weight distribution was ranging from -0.9 to -0.4 .

Analysis of disruptions in CLL modules. On the basis of the threshold of nodes $\geq 5$, there were 8,773 maximal cliques in the normal and CLL PPI sub-networks, and then, these 8,773 cliques were utilized to conduct module analysis. There were 1,805 and 703 modules from the PPI sub-networks of normal and CLL, respectively (Table I). For the module of CLL, the average module size was higher than that of module of normal. Moreover, for CLL modules, it also showed an overall decrease in average correlation comparing with the normal modules. The connection between the counts of modules and weighted density of modules is shown in Fig. 2. The distribution of modules in normal was higher under $0-0.2$, while lower under $0.2-0.8$ relative to that in disease condition.

Moreover, there were 646 disrupted module pairs. Relative to the normal group, we found that all altered modules were higher in the disease group based on the module correlation density of the modules.

Gene composition of the altered modules. There were 646 disrupted module pairs in total. Among these module pairs, there were 145 missed genes and 353 added genes in CLL modules relative to normal modules. Of note, 85 genes were the common ones between the added and missed genes. In this investigation, we obtained 9 common genes between the 85 intersection genes and the DEGs, of which were AKT1, UPF3A, SMG7, RPN2, HSPB1, SPCS2, CDC16, COPS5 and PSMB10. Hence, we treated the 9 genes as the key genes involved in CLL.

\section{Discussion}

CLL is a variety of chronic lymphoproliferative disorders (24). In our study, we investigated gene profile E-GEOD-2466 and extracted the key genes in CLL on the basis of systematically tracking the disrupted modules of re-weighted PPI networks. In total, 9 key genes were identified through comparing intersection genes in the altered modules and DEGs.

In humans, RAC- $\alpha$ serine/threonine-protein kinase is coded by AKT1 gene. AKT1 has been demonstrated to regulate a large amount of biological processes, for example, cell proliferation, growth, metabolism, survival, and angiogenesis. The AKT1 isoform played a dominant role in the survival and chemoresistance of CLL cells (25). AKT1 gene has been inferred to be related to a variety of diseases. It was familiar in cancer $(26,27)$, tumors $(28,29)$ and insulin resistance $(30)$. Another study has also implicated that the expression of AKT1 was associated with CLL $(31,32)$.

Dolichyl-diphosphooligosaccharide-protein glycosyltransferase subunit 2 is coded by RPN2 in humans. So far there has not been much research on the correlation of RPN2 and a disease, in addition to tumors. For example, it has been verified that RPN2 silencing made breast cells hypersensitive to docetaxel (33). Moreover, RPN2 has also displayed a higher genomic alteration frequency in colorectal cancer (34).

Heat-shock protein 27 (Hsp27) in humans is encoded by HSPB1, which is a chaperone of the small heat-shock protein group $(35,36)$. As known, the common roles of small heat-shock proteins are inhibition of apoptosis, thermotolerance, and cell differentiation. HSPB1 was reported related to breast cancer, shock, Alexander's disease and many kinds of tumors (37-40). Dempsey et al (41) have explored that CLL patients expressed significantly higher levels of Hsp27 in lymphocytes than that expressed by lymphocytes from control subjects.

Taken together, we successfully identified a series of key genes for understanding the potential mechanism of gene expression of CLL on the basis of systematically uncovering the altered modules based on the re-weighted PPI networks and DEGs. These results have implications for understanding the mechanisms of gene expression of CLL.

\section{Acknowledgements}

Not applicable.

\section{Funding}

No funding was received. 


\section{Availability of data and materials}

The datasets used and/or analyzed during the current study are available from the corresponding author on reasonable request.

\section{Authors' contributions}

$\mathrm{XL}$ and SFK conceived the study and drafted the manuscript. $\mathrm{ZW}$ and XYZ acquired the data. CFL and SFK analyzed the data and revised the manuscript. All authors read and approved the final manuscript.

\section{Ethics approval and consent to participate}

Not applicable.

\section{Patient consent for publication}

Not applicable.

\section{Competing interests}

The authors declare that they have no competing interests.

\section{References}

1. Nguyen PV, Srihari S and Leong HW: Identifying conserved protein complexes between species by constructing interolog networks. BMC Bioinformatics 14 (Suppl 16): S8, 2013.

2. Srihari S and Leong HW: A survey of computational methods for protein complex prediction from protein interaction networks. J Bioinform Comput Biol 11: 1230002, 2013.

3. Li X, Wu M, Kwoh CK and Ng SK: Computational approaches for detecting protein complexes from protein interaction networks: A survey. BMC Genomics 11 (Suppl 1): S3, 2010.

4. Bader GD and Hogue CW: An automated method for finding molecular complexes in large protein interaction networks. BMC Bioinformatics 4: 2, 2003.

5. Liu G, Wong L and Chua HN: Complex discovery from weighted PPI networks. Bioinformatics 25: 1891-1897, 2009.

6. Hwang W, Cho YR, Zhang A and Ramanathan M: A novel functional module detection algorithm for protein-protein interaction networks. Algorithms Mol Biol 1: 24, 2006.

7. Srihari S and Ragan MA: Systematic tracking of dysregulated modules identifies novel genes in cancer. Bioinformatics 29: $1553-1561,2013$.

8. Byrd JC, Stilgenbauer S and Flinn IW: Chronic lymphocytic leukemia. Hematology (Am Soc Hematol Educ Program) 2004: 163-183, 2004.

9. Abrisqueta P, Pereira A, Rozman C, Aymerich M, Giné E, Moreno C, Muntañola A, Rozman M, Villamor N, Hodgson $\mathrm{K}$, et al: Improving survival in patients with chronic lymphocytic leukemia (1980-2008): The Hospital Clinic of Barcelona experience. Blood 114: 2044-2050, 2009.

10. Rawstron AC, Böttcher S, Letestu R, Villamor N, Fazi C, Kartsios H, de Tute RM, Shingles J, Ritgen M, Moreno C, et al; European Research Initiative in CLL: Improving efficiency and sensitivity: European Research Initiative in CLL (ERIC) update on the international harmonised approach for flow cytometric residual disease monitoring in CLL. Leukemia 27: 142-149, 2013.

11. van den Broek EC, Kater AP, van de Schans SA, Karim-Kos HE, Janssen-Heijnen ML, Peters WG, Nooijen PT, Coebergh JW and Posthuma EF: Chronic lymphocytic leukaemia in the Netherlands: Trends in incidence, treatment and survival, 1989-2008. Eur J Cancer 48: 889-895, 2012.

12. Rozovski U, Hazan-Halevy I, Keating MJ and Estrov Z: Personalized medicine in CLL: Current status and future perspectives. Cancer Lett 352: 4-14, 2014.

13. Cavallini C, Lovato O, Bertolaso A, Zoratti E, Malpeli G, Mimiola E, Tinelli M, Aprili F, Tecchio C, Perbellini O, et al: Expression and function of the TL1A/DR3 axis in chronic lymphocytic leukemia. Oncotarget 6: 32061-32074, 2015.
14. Montresor A, Toffali L, Mirenda M, Rigo A, Vinante F and Laudanna C: JAK2 tyrosine kinase mediates integrin activation induced by CXCL12 in B-cell chronic lymphocytic leukemia. Oncotarget 6: 34245-34257, 2015.

15. Haslinger C, Schweifer N, Stilgenbauer S, Döhner H, Lichter P, Kraut N, Stratowa C and Abseher R: Microarray gene expression profiling of B-cell chronic lymphocytic leukemia subgroups defined by genomic aberrations and VH mutation status. J Clin Oncol 22: 3937-3949, 2004.

16. Irizarry RA, Bolstad BM, Collin F, Cope LM, Hobbs B and Speed TP: Summaries of Affymetrix GeneChip probe level data. Nucleic Acids Res 31: e15-e15, 2003.

17. Bolstad BM, Irizarry RA, Astrand M and Speed TP: A comparison of normalization methods for high density oligonucleotide array data based on variance and bias. Bioinformatics 19: 185-193, 2003.

18. Bolstad B: affy: Built-in processing methods. https://www. bioconductor.org/packages/3.7/bioc/vignettes/affy/inst/doc/builtinMethods.pdf. Accessed 5, 10, 2017.

19. Smyth GK, Ritchie M, Thorne N, Wettenhall J and Shi W: limma: Linear models for microarray data user's guide. http://citeseerx. ist.psu.edu/viewdoc/download?doi=10.1.1.361.8519\&rep=rep1\& type $=$ pdf. Accessed 5, 10, 2017.

20. Datta S, Satten GA, Benos DJ, Xia J, Heslin MJ and Datta S: An empirical bayes adjustment to increase the sensitivity of detecting differentially expressed genes in microarray experiments. Bioinformatics 20: 235-242, 2004.

21. Reiner A, Yekutieli D and Benjamini Y: Identifying differentially expressed genes using false discovery rate controlling procedures. Bioinformatics 19: 368-375, 2003.

22. von Mering C, Jensen LJ, Snel B, Hooper SD, Krupp M, Foglierini M, Jouffre N, Huynen MA and Bork P: STRING: Known and predicted protein-protein associations, integrated and transferred across organisms. Nucleic Acids Res 33: D433-D437, 2005.

23. Hodge JC, Kim T-M, Dreyfuss JM, Somasundaram P Christacos NC, Rousselle M, Quade BJ, Park PJ, Stewart EA and Morton CC: Expression profiling of uterine leiomyomata cytogenetic subgroups reveals distinct signatures in matched myometrium: Transcriptional profiling of the $t(12 ; 14)$ and evidence in support of predisposing genetic heterogeneity. Hum Mol Genet 21: 2312-2329, 2012.

24. Kayar Y, Ekinci I, Bay I, Bayram Kayar N, Hamdard J and Kazancıoğlu R: Acute renal failure due to leukaemic infiltration in chronic lymphocytic leukaemia. Case Rep Med 2015: 469136 , 2015.

25. Hof bauer SW, Krenn PW,Piñón Hofbauer J,Pucher S, Asslaber D, Egle A, Hartmann TN and Greil R: The AKT1 isoform plays a dominant role in the survival and chemoresistance of chronic lymphocytic leukaemia cells. Br J Haematol 172: 815-819, 2016.

26. Carson JP, Kulik G and Weber MJ: Antiapoptotic signaling in LNCaP prostate cancer cells: A survival signaling pathway independent of phosphatidylinositol 3'-kinase and Akt/protein kinase B. Cancer Res 59: 1449-1453, 1999.

27. Kim D, Kim S, Koh H, Yoon SO, Chung AS, Cho KS and Chung J: Akt/PKB promotes cancer cell invasion via increased motility and metalloproteinase production. FASEB J 15: 1953-1962, 2001.

28. Goldstein M, Meller I and Orr-Urtreger A: FGFR1 over-expression in primary rhabdomyosarcoma tumors is associated with hypomethylation of a 5' $\mathrm{CpG}$ island and abnormal expression of the AKT1, NOG, and BMP4 genes. Genes Chromosomes Cancer 46: 1028-1038, 2007.

29. Ericson K, Gan C, Cheong I, Rago C, Samuels Y, Velculescu VE, Kinzler KW, Huso DL, Vogelstein B and Papadopoulos N: Genetic inactivation of AKT1, AKT2, and PDPK1 in human colorectal cancer cells clarifies their roles in tumor growth regulation. Proc Natl Acad Sci USA 107: 2598-2603, 2010.

30. Cleasby ME, Reinten TA, Cooney GJ, James DE and Kraegen EW: Functional studies of Akt isoform specificity in skeletal muscle in vivo; maintained insulin sensitivity despite reduced insulin receptor substrate-1 expression. Mol Endocrinol 21: 215-228, 2007.

31. Zhuang J, Hawkins SF, Glenn MA, Lin K, Johnson GG, Carter A, Cawley JC and Pettitt AR: Akt is activated in chronic lymphocytic leukemia cells and delivers a pro-survival signal: The therapeutic potential of Akt inhibition. Haematologica 95: $110-118,2010$.

32. de Frias M, Iglesias-Serret D, Cosialls AM, Coll-Mulet L, Santidrián AF, González-Gironès DM, de la Banda E, Pons G and Gil J: Akt inhibitors induce apoptosis in chronic lymphocytic leukemia cells. Haematologica 94: 1698-1707, 2009. 
33. Honma K, Iwao-Koizumi K, Takeshita F, Yamamoto Y, Yoshida T, Nishio K, Nagahara S, Kato K and Ochiya T: RPN2 gene confers docetaxel resistance in breast cancer. Nat Med 14: 939-948, 2008.

34. Zhang J, Yan B, Späth SS, Qun H, Cornelius S, Guan D, Shao J, Hagiwara K, Van Waes C, Chen Z, et al: Integrated transcriptional profiling and genomic analyses reveal RPN2 and HMGB1 as promising biomarkers in colorectal cancer. Cell Biosci 5: 53, 2015.

35. Carper SW, Rocheleau TA and Storm FK: cDNA sequence of a human heat shock protein HSP27. Nucleic Acids Res 18: 6457, 1990.

36. Hunt CR, Goswami PC and Kozak CA: Assignment of the mouse Hsp25 and Hsp105 genes to the distal region of chromosome 5 by linkage analysis. Genomics 45: 462-463, 1997.

37. Shi P, Wang M-M, Jiang L-Y, Liu H-T and Sun J-Z: Paclitaxel-doxorubicin sequence is more effective in breast cancer cells with heat shock protein 27 overexpression. Chin Med J (Engl) 121: 1975-1979, 2008.
38. Shen G, Liang S, Xu Z, Zhou L, Xiao S, Xia X, Li R, Liao Y, You C and Wei Y: Downregulated expression of HSP27 in human low-grade glioma tissues discovered by a quantitative proteomic analysis. Proteome Sci 8: 17, 2010.

39. Iwaki T, Iwaki A, Tateishi J, Sakaki Y and Goldman JE: Alpha B-crystallin and 27-kd heat shock protein are regulated by stress conditions in the central nervous system and accumulate in Rosenthal fibers. Am J Pathol 143: 487-495, 1993.

40. Wu Y, Liu J, Zhang Z, Huang H, Shen J, Zhang S, Jiang Y, Luo L and Yin Z: HSP27 regulates IL-1 stimulated IKK activation through interacting with TRAF6 and affecting its ubiquitination. Cell Signal 21: 143-150, 2009.

41. Dempsey NC, Leoni F, Ireland HE, Hoyle C and Williams JH: Differential heat shock protein localization in chronic lymphocytic leukemia. J Leukoc Biol 87: 467-476, 2010.

This work is licensed under a Creative Commons Attribution-NonCommercial-NoDerivatives 4.0 International (CC BY-NC-ND 4.0) License. 